\title{
FOUR DIFFERENT APPROACHES TO THE NORMALIZED BANZHAF VALUES OF GAMES WITH A PRIORI UNIONS
}

\author{
HONORATA SOSNOWSKA \\ Department of Mathematical Economics, Warsaw School of Economics \\ Al. Niepodległości 162, 02-554 Warszawa, Poland \\ E-mail: honorata@sgh.waw.pl
}

\begin{abstract}
Applying the Owen construction of value of games with a priori unions to the normalized Banzhaf value gives a new type of the normalized Banzhaf value for games with a priori unions. Using a simple example of a four-person voting game with a priori unions, it is shown that this value is different from those known in the literature: the normalized Owen-Banzhaf value, the Banzhaf share function defined by van der Laan and van den Brink and the Banzhaf index for simple games with a priori unions introduced by Malawski. Thus, all four notions are distinct.
\end{abstract}

1. Introduction. The standard notion of value of cooperative games does not describe the situation where some players prefer to act together. To deal with such situations, Owen (1977) introduced the notion of game with a priori unions and defined the Shapley value and later (1981) also the Banzhaf value for games with a priori unions. His construction of values of games with a priori unions can be applied to any value defined for all cooperative games (cf. Młodak 2003). In this paper we apply it to the normalized Banzhaf value.

A question arises if the value for games with a priori unions which obtains from this construction coincides with the normalized Owen-Banzhaf value obtained by normalizing the Owen's extension of the Banzhaf value. We demonstrate, using an example of a fourperson weighted majority voting game with an appropriate a priori unions structure, that the answer is negative. Moreover, the same example lets us compare our notion to two other approaches to the normalized Banzhaf value of games with a priori unions: the Banzhaf coalition structure share functions for monotone games, defined by van der Laan and van den Brink (2002), and the Banzhaf index for simple games with a priori unions defined by Malawski (2004).

The game in this example is both simple and monotone, and its normalized OwenBanzhaf value is equal to both its Banhaf share function and to its Banzhaf index. Thus,

2000 Mathematics Subject Classification: Primary 91A12.

The paper is in final form and no version of it will be published elsewhere. 
the Owen construction applied to the normalized Banhaf value yields a value different from all three previously known and, since it is known that those three approaches led to three different normalized values, we conclude that all four values are different even for monotone simple games.

In the next section we introduce the basic notions, the general Owen's construction of value of games with a priori unions and four possible definitions of normalized Banzhaf value for games with a priori unions. The main example is analyzed in section 3 . The last section is devoted to conclusions.

2. Values of games with a priori unions. An $n$-person game on the player set $N=\{1,2, \ldots, n\}$ is a function $v: 2^{N} \rightarrow \mathbb{R}$ satisfying $v(\emptyset)=0$. Let $T=\left(T_{1}, \ldots, T_{m}\right)$ be a partition of the set $N$ into coalitions which are nonempty, pairwise disjoint and their union is $N$. The coalitions $T_{j}, j=1, \ldots, m$ are called a priori unions or precoalitions. Denote the set of a priori unions by $M=\{1, \ldots, m\}$.

The pair $(v, T)$ is called a game with a priori unions. Every such game determines the following new games:

- the quotient game $u=v / T$ with the player set $M$ :

$$
u(S)=v\left(\bigcup_{j \in S} T_{j}\right), \quad \text { where } S \subseteq M
$$

(the game played among the a priori unions),

- the family of altered games: for every $j \in M$ and every $K \subseteq T_{j}, u_{T_{j}, K}$ is the game on $M$ given by.

$$
u_{T_{j}, K}(S)=u(S) \quad \text { if } j \notin S
$$

and

$$
u_{T_{j}, K}=v\left(K \cup \bigcup_{l \in S \backslash\{j\}} T_{l}\right) \quad \text { if } j \in S .
$$

- that is, $T_{j}$ gets replaced by $K$ in the quotient game.

2.1. Extending values to games with a priori unions. Recall that a value is any mapping $q$ from the set (or subset) of cooperative games to $\bigcup_{k=1}^{\infty} \mathbb{R}^{k}$ such that for any $n$-person game $v$ in the domain of $q, q(v)=\left(q_{1}(v), \ldots, q_{n}(v)\right) \in \mathbb{R}^{n}$. The following construction is a generalization of Owen's construction of Shapley value for games with a priori unions to any value for cooperative games:

Given a value $q$ and a game with a priori unions $(v, T)$, define the reduced game $w_{j}$ on $T_{j}$ by

$$
w_{j}(K)=q_{j}\left(u_{T_{j}, K}\right)
$$

for every $j=1, \ldots, m$ and any subset $K \subseteq T_{j}$, and the value of the game with a priori unions, $q(v, T)$, by

$$
q_{i}(v, T)=q_{i}\left(w_{j}\right), \quad i \in T_{j}, i=1, \ldots, n, j=1, \ldots, m .
$$

That is, the value of player $i$ is his value in the reduced game of the a priori union to which $i$ belongs. 
2.2. Two types of normalized Banzhaf value based on Owen's construction. Let $v$ be an $n$-person cooperative game. The Banzhaf value (Banzhaf,1965) of $v$ is defined by the formula

$$
B_{i}(v)=\frac{\sum_{K \subseteq N}[v(K \cup\{i\})-v(K)]}{2^{n-1}} .
$$

This is also known as the absolute Banzhaf value.

Owen (1981) extended the absolute Banzhaf value to games with a priori unions by the following formula:

$$
B_{i}(v, T)=2^{1-m} 2^{1-t_{j}} \sum_{S \subseteq M} \sum_{K \subseteq T_{j}}\left[v\left(Q_{S} \cup K \cup\{i\}\right)-v\left(Q_{S} \cup K\right)\right],
$$

where $T_{j} \ni i, t_{j}=\# T_{j}, Q_{S}=\bigcup_{r \in S} T_{r}$ and $i=1, \ldots, n$. This extension is now known as the Owen-Banzhaf value.

While both the Banzhaf value and the Owen-Banzhaf value are defined for all cooperative games, their normalized versions can only be defined for games in which the sum of the value's components is not equal to 0 . Therefore, throughout the rest of the paper we restrict our attention to monotone games and define all normalized values only for such games. The game $v$ is monotone if and only if

$$
S \subseteq T \Rightarrow v(S) \leq v(T) .
$$

The Banzhaf value and the Owen-Banzhaf value are not normalized: the sum of values of all players may be different from $v(N)$. Normalizing them for non-null monotone games leads to the relative, or normalized Banzhaf value $B Z$ :

$$
B Z_{i}(v)=\frac{B_{i}(v) \cdot v(N)}{\sum_{i=1}^{n} B_{i}(v)}
$$

and to the first type of normalized Banzhaf value of games with a priori unions, $B N$ (obtained by normalizing the Owen-Banzhaf value):

$$
B N_{i}(v, T)=\frac{B_{i}(v, T)}{\sum_{i=1}^{n} B_{i}(v, T)} \cdot v(N) .
$$

For the null game $v^{0}$ (i.e., $v^{0}(E)=0$ for every $E \subseteq N$ ), we define

$$
B Z_{i}\left(v^{0}\right)=B N_{i}\left(v^{0}, T\right)=0, \quad i=1, \ldots, n .
$$

However, a normalized Banzhaf value of (monotone) games with a priori unions may also be defined in another way - by applying the Owen construction to the normalized Banzhaf value, as proposed in Młodak (1999). We thus obtain the second type of the normalized Banzhaf value and denote it by $B Z_{i}(v, T)$. The exact formula is very complicated but we shall not need it in what follows.

2.3. The Banzhaf coalition structure share function. Van der Laan and van den Brink (2002) introduced the Banzhaf share function and the Banzhaf coalition structure share function. Let $v$ be a monotone $n$-person game. The Banzhaf share function $r^{B}$ of a game $v$ is given by

$$
\begin{aligned}
r_{i}^{B}(v)=B Z_{i}(v) / v(N) & \text { for } v \neq v^{0}, \\
r_{i}^{B}\left(v^{0}\right)=1 / n, & i=1, \ldots, n .
\end{aligned}
$$


The Banzhaf coalition structure share function is defined in the following way. Consider an $n$-person game $v$ on $N=\{1, \ldots, n\}$ and a partition $T=\left\{T_{1}, \ldots, T_{m}\right\}$ of set $N$. The first level game $v^{T}$ on the set $M=\{1, \ldots, m\}$ of a priori unions is defined by

$$
v^{T}(L)=v(P(L))
$$

for $L \subseteq M$ and $P(L)=\bigcup_{j \in L} T_{j}$. So, $v^{T}$ is exactly the quotient game $u$ defined by formula (1). Then for every $T_{k}$ and every $L \subset M, k \notin L$ we define the game $v^{k, L}$ on $T_{k}$ :

$$
v^{k, L}(E)=v(E \cup P(L))-v(P(L)), \quad E \subseteq T_{k} .
$$

Then the second level game $v^{k}$ on $T_{k}$ is given by the formula

$$
v^{k}(E)=\sum_{L \subset M, k \notin L} \frac{v^{k, L}(E)}{2^{m-1}}, \quad E \subseteq T^{k} .
$$

The Banzhaf coalition structure share function is defined by the equation

$$
B L_{i}(v, T)=r_{i}^{B}\left(v^{k}\right) \cdot r_{k}^{B}\left(v^{T}\right), \quad i \in T_{k} .
$$

Van der Laan and van den Brink (2002) proved the following properties of the Banzhaf coalition structure share function:

THEOREM 2.1. (i) $\sum_{i \in N} B L_{i}(v, T)=1$ (efficiency).

(ii) (a) $\sum_{i \in T_{k}} B L_{i}(v)=r_{k}^{B}\left(v^{T}\right)$ and

(b) if $T=\{N\}$ or $T=\{\{i\}, i=1, \ldots, n\}$, then $B L_{i}(v, T)=r_{i}^{B}(v)$ (consistency).

(iii) Let $v$ be a simple game and $T=\{C,\{h\}, h \in N-C\}$, where $C$ is a majority coalition. Then

$$
B L_{i}(v, T)=r_{i}^{B}(v) / \sum_{j \in C} r_{j}^{B}(v) \quad \text { if } i \in C \quad \text { and } \quad B L_{i}(v, T)=0 \text { if } i \notin C .
$$

2.4. The counting Banzhaf index with a priori unions. Malawski (2004) studied a class of counting power indices for simple games; the normalized Banzhaf index belongs to this class.

Let $N=\{1, \ldots, n\}$ be a set of players. A monotone game $v: 2^{N} \rightarrow\{0,1\}$ such that $v(\emptyset)=0, v(N)=1$ is called a simple $n$-person game. In this section we shall consider simple games only. For a player $k$ in a simple game $v$ denote

$$
D(k, v)=\{U \subseteq N: v(U)=1 \text { and } v(U-\{k\})=0\} .
$$

A power index is any function $p$ which assigns to each $n$-person simple game a vector $p(v) \in \mathbb{R}^{n}$ such that $\sum_{i=1}^{n} p_{i}(v)=1$ and $0 \leq p_{i}(v) \leq 1, i=1, \ldots n$. A power index is called a counting index if it is of the form

$$
p_{j}(v)=\frac{\sum_{S \ni j} c_{v}(S)(v(S)-v(S \backslash\{j\})}{\sum_{k=1}^{n} \sum_{T \ni k} c_{v}(T)(v(T)-v(T \backslash\{k\}))}=\frac{\sum_{S \in D(j, v)} c_{v}(S)}{\sum_{k=1}^{n} \sum_{T \in D(k, v)} c_{v}(T)}
$$

where the coefficients $c_{v}(T)$ are nonnegative and depend only on the restriction of $v$ to $T$. For the normalized Banzhaf index $c_{v}(T) \equiv 1$.

To extend any counting index to simple games with a priori unions, take any such game $(v, T)$ and consider the quotient game $u$ on $M$ defined by equation (1). Let us also 
fix a player $j \in M$ in this quotient game. For every coalition $W \subseteq M$ such that $u(W)=1$ and $u(W-\{j\})=0$ (that is, $W \in D(j, u)$ ) we define a game $v_{j, W}$ on $T_{j}$ by

$$
v_{j, W}(S)=v\left(\bigcup_{l \in W \backslash\{j\}} T_{l} \cup S\right), \quad S \subseteq T_{j} .
$$

Now, given an index $p$ for simple games, we compute a "pre-index"

$$
p_{i}^{*}(v, T)=\sum_{W \in D(j, u)} c_{u}(j, W) \cdot p_{i}\left(v_{j, W}\right), \quad i \in T_{j},
$$

and normalize it to obtain a counting index with a priori unions:

$$
p_{i}(v, T)=p_{i}^{*}(v, T) / \sum_{k=1}^{n} p_{k}^{*}(v, T)
$$

We shall denote the normalized counting Banzhaf index with a priori unions by $B M$. It is given by the formula

$$
B M_{i}(v, T)=\frac{\sum_{W \in D(j, u)}\left(\# D\left(i, v_{j, W}\right) / \sum_{k \in T_{j}} \# D\left(k, v_{j, W}\right)\right)}{\sum_{k=1}^{n} \sum_{Y \in D(l, u)}\left(\# D\left(k, v_{l, Y}\right) / \sum_{m \in T_{l}} \# D\left(m, v_{l, Y}\right)\right)}
$$

where $T_{j} \ni i$ and $T_{l} \ni k$.

3. Comparing the normalized values. We first check if

$$
B N(v, T)=B Z(v, T) .
$$

We need weighted majority voting games to answer the question. An $n$-person weighted majority voting game is determined by $n$ (nonnegative) weights of players, $d_{1}, \ldots, d_{n}$, and a positive number $d \leq \sum_{i=1}^{n} d_{i}$ denoting the minimum winning majority. The game is defined as follows: for any coalition $K \subseteq N, v(K)=1$ if and only if $\sum_{i \in K} d_{i} \geq d$. Otherwise $v(K)=0$.

A weighted majority voting game is denoted by $v=\left(d ; d_{1}, \ldots, d_{n}\right)$. Obviously, all majority voting games are simple games.

THEOREM 3.1. There exists a weighted majority voting game $v$ and a partition $T$ such that $\left.B N(v, T) \neq B Z_{(} v, T\right)$.

Proof. Let us consider the 4-person game $v=(5 ; 4,2,2,2)$ and the partition $T=$ $\{\{1,2\},\{3\},\{4\}\}$. We compute $B Z(v, T)$ and $B N(v)$.

i) The quotient game is $U=(5 ; 6,2,2)$, so $B(u)=B Z(u)=(1,0,0)$.

ii) (a) The altered games for $T_{1}=\{1,2\}$ are:

$$
\begin{aligned}
& u_{T_{1}, \emptyset}=(5 ; 0,2,2) \text { - the null game; } \\
& u_{T_{1},\{1\}}=(5 ; 4,2,2) \text {, so } \\
& B\left(u_{T_{1},\{1\}}\right)=(3 / 4,1 / 4,1 / 4) \text { and } B Z\left(u_{T_{1},\{1\}}\right)=(3 / 5,1 / 5,1 / 5) ; \\
& u_{T_{1},\{2\}}=(5 ; 2,2,2) \text {, so } \\
& B\left(u_{T_{1},\{2\}}\right)=(1 / 4,1 / 4,1 / 4) \text { and } B Z\left(u_{T_{1},\{2\}}\right)=(1 / 3,1 / 3,1 / 3) ;
\end{aligned}
$$

and $u_{T_{1}, T_{1}}=u$. 
(b) The altered games for $T_{2}=\{3\}$ are

$$
\begin{aligned}
u_{T_{2}, \emptyset}=(5 ; 6,0,2) & \text { and } u_{T_{2}, T_{2}}=u \text {, so } \\
& B\left(u_{T_{2}, \emptyset}\right)=B Z\left(u_{T_{2}, \emptyset}\right)=B\left(u_{T_{2}, T_{2}}\right)=B Z\left(u_{T_{2}, T_{2}}\right)=(1,0,0) .
\end{aligned}
$$

Analogously, we get the same values for the altered games for $T_{2}=\{3\}$.

Therefore, the reduced games $w_{2}$ and $w_{3}$ are null games.

iii) The reduced games on $T_{1}$ are:

the game $w_{1}$ based on the Banzhaf value:

$$
\begin{aligned}
& \left.w_{1}(\emptyset)=0\right), w_{1}(\{1\})=\left(B_{1}\left(u_{T_{1},\{1\}}\right)=3 / 4,\right. \\
& w_{1}(\{2\})=B_{1}\left(u_{T_{1},\{2\}}\right)=1 / 4, w_{1}\left(T_{1}\right)=B_{1}(u)=1, \\
& \text { and so } B\left(w_{1}\right)=B Z\left(w_{1}\right)=(3 / 4,1 / 4)
\end{aligned}
$$

the game $w_{1}^{\prime}$ based on the normalized Banzhaf value:

$$
\begin{aligned}
& w_{1}^{\prime}(\emptyset)=0, w_{1}^{\prime}(\{1\})=B Z_{1}\left(u_{T_{1},\{1\}}\right)=3 / 5, \\
& w_{1}^{\prime}(\{2\})=B Z_{1}\left(u_{T_{1},\{2\}}\right)=1 / 3, w_{1}^{\prime}(\{1,2\})=B Z_{1}(u)=1 \\
& \text { and so } B\left(w_{1}^{\prime}\right)=B Z\left(w_{1}^{\prime}\right)=(19 / 30,11 / 30) .
\end{aligned}
$$

iv) Together, the values of reduced games give

$$
B N(v, T)=B(v, T)=(3 / 4,1 / 4,0,0)
$$

and

$$
B Z(v, T)=(19 / 30,11 / 30,0,0) .
$$

Thus, $B N(v, T) \neq B Z(v, T)$.

The above example shows that the two types of the normalized Banzhaf value of a game with a priori unions are different. The same example can also be used for comparing $B Z$ with $B L$ and $B M$.

It is obvious that in general $B L \neq B N$ and $B L \neq B Z$, since the components of $B L$, a share function, always add to 1 while those of $B N$ and $B Z$, the normalized values, add to $v(N)$. However, it makes sense to ask whether some of the two above values equals $B L$ on the class of simple games (or, more generally, whether it equals $B L \cdot v(N)$ for monotone games). The answer is negative. Van den Brink and van der Laan (2002) explicitly proved that $B L \neq B N \cdot v(N)$. Moreover, the (simple) game $v=(5 ; 4,2,2,2)$ and the partition $T$ of example satisfy the assumptions of theorem 2.1 (iii), so $B L(v, T) \cdot v(N)=(3 / 4 \cdot 1,1 / 4$. $1,0,0) \cdot 1 \neq B Z(v, T)$.

Also, Malawski (2004, example 1) computed that for the majority voting game $w=$ $(55 ; 40,20,20,20) B M(v, T)=(3 / 4,1 / 4,0,0)$. The game $w$ is equivalent to $v$, so $B M(v, T)$ $=B M(w, T) \neq B Z(v, T)$. Moreover, a direct consequence of theorem 1 in Malawski (2004) is that $B M \neq B N$ on the domain of $B M$.

Finally, since the Banzhaf coalition structure share function $B L$ has the multiplication property (15), it follows that it is different from the counting Banzhaf index with a priori unions for some simple games. Combining the above statements, we obtain

Corollary. All the values $B N, B Z, B L$ and $B M$ on the class of simple games are different. 
4. Concluding remarks. We have formally shown that four ways of constructing the normalized Banzhaf value of games with a priori unions lead to four values which are indeed different. We have deliberately avoided the questions of intuitions behind, and interpretations of these notions. Nevertheless, the fact that they all differ even on the class of monotone simple games (which is the intersection of their domains) suggests that the very idea of the normalized Banzhaf value of games with a priori unions relies rather on formal construction than on sound intuition.

A sharp contrast between the Banzhaf value and the Shapley value is worth mentioning here: for the Shapley value, all four constructions lead to the same value with a priori unions (some trivially, because the value itself is normalized), which, moreover, has a clear probabilistic interpretation.

\section{References}

[1] J. F. Banzhaf III, Weighted voting doesn't work: a mathematical analysis, Rutgers Law Review 19 (1965), 317-343.

[2] M. Malawski, 'Counting' power indices for games with a priori unions, Theory and Decision 56 (2004), 125-140.

[3] A. Młodak, Some values of game with a priori unions (in Polish), doctoral thesis, Warsaw School of Economics, Warsaw, 1999.

[4] A. Młodak, Three additive solutions of cooperative games with a priori unions, Applicationes Mathematicae 30 (2003), 69-87.

[5] G. Owen, Values of games with a priori unions, in: Mathematical Economics and Game Theory, Henn and Moeschlin (eds.), Lecture Notes in Economics and Mathematical Sysytems 141, Springer, 1977, 76-88.

[6] G. Owen, Modification of the Banzhaf-Coleman index fot games with a priori unions, in: Power, Voting and Voting Power, M. J. Holler (ed.), Physica Verlag, Würzburg, 1981, $355-356$.

[7] G. van der Laan and R. van den Brink, A Banzhaf share function for cooperative games in coalition structure, Theory and Decision 53 (2002), 61-86. 